\title{
Correlations in hot asymmetric nuclear matter
}

\author{
T. Frick and H. Müther \\ Institut für Theoretische Physik, Universität Tübingen, D-72076 Tübingen, Germany \\ A. Rios, A. Polls, and A. Ramos \\ Departament d'Estructura i Constituents de la Matèria, Universitat de Barcelona, E-08028 Barcelona, Spain
}

(Received 28 September 2004; published 27 January 2005)

\begin{abstract}
The single-particle spectral functions in asymmetric nuclear matter are computed using the ladder approximation within the theory of finite temperature Green's functions. The internal energy and the momentum distributions of protons and neutrons are studied as a function of the density and the asymmetry of the system. The proton states are more strongly depleted when the asymmetry increases whereas the occupation of the neutron states is enhanced compared to the symmetric case. The self-consistent Green's function approach leads to slightly smaller energies compared to the Brueckner-Hartree-Fock approach. This effect increases with density and thereby modifies the saturation density and leads to smaller symmetry energies.
\end{abstract}

DOI: 10.1103/PhysRevC.71.014313

PACS number(s): 21.65.+f, 21.30.Fe

\section{INTRODUCTION}

The equation of state (EOS) of asymmetric nuclear matter is a necessary ingredient in the description of astrophysical environments of interest such as supernova explosions or the structure of neutron stars [1]. Actually, the study of asymmetric nuclear matter is also relevant to understand stable nuclei because they themselves are asymmetric nuclear systems with a different number of protons and neutrons. The recent availability of data concerning nuclei far from the stability valley has also revitalized interest in the study of asymmetric nuclear matter as a first step in the microscopic study of these nuclei.

The evaluation of this EOS starting from realistic models of the nucleon-nucleon $(N N)$ interaction is still one of the challenging open problems in nuclear physics. In fact, the presence of strong short-range and tensor components in the realistic $N N$ interactions, which are required to fit the $N N$ scattering data, are the origin of the corresponding correlations in the nuclear wave function. The study of these correlations and their influence on different observables has recently made important progress not only from the theoretical side but also from the experimental point of view [2-4]. In the latter case, the analysis of $\left(e, e^{\prime} p\right)$ reactions on ${ }^{208} \mathrm{~Pb}$ covering a wide range of missing energies led to the conclusion that the occupation numbers for the deeply bound proton states are depleted by about $15-20 \%$ [5]. This depletion can be identified with the corresponding depletion of hole states in nuclear matter with momenta well below the Fermi momentum [6-8].

Several theoretical tools have been developed and applied to describe these correlations in nuclear systems. These include the Brueckner hole-line expansion [9] and also variational approaches using correlated basis functions [10-12].

Recently, enormous progress has been achieved in using the self-consistent evaluation of Green's function [13,14] to solve the nuclear many-body problem [3,4,15-20]. This method gives direct access to the single-particle spectral function, that is, to the single-particle properties and, in particular, to the occupation numbers. At the same time, modification of the single-particle properties affects the effective interactions among nucleons in the medium and both things should be determined in a self-consistent way.

Most of the microscopic calculations have been addressed to study symmetric nuclear matter (SNM) and pure neutron matter (PNM) [16,17]. The study of asymmetric nuclear matter is technically more involved and only a few Brueckner-Hartree Fock (BHF) calculations are available [21-23]. In most of the cases, one assumes a quadratic dependence of the energy per particle,

$$
\frac{E}{A}(\rho, \alpha)=\frac{E}{A}(\rho, 0)+a_{s}(\rho) \alpha^{2}+\ldots,
$$

in terms of the asymmetry parameter $\alpha=(N-Z) / A$ and the symmetry energy $a_{s}(\rho)$. In this way, calculation of the energy of SNM and PNM allows one to determine the symmetry energy $a_{s}(\rho)$ and using the previous equation one can estimate the energy for any asymmetry. That this quadratic expression is a good approximation has been directly confirmed in BHF calculations of asymmetric nuclear matter [21,22]. In the case of the variational approach, calculations for asymmetric matter with the same accuracy as obtained for SNM or PNM are not yet available. However, besides the energy per particle, which is governed mainly by the symmetry energy, there are other observables, such as the momentum distributions, that do not necessary follow a quadratic dependence.

In this paper we want to perform a calculation of asymmetric nuclear matter within the framework of SCGF theory. The calculation is performed at finite temperature. A temperature of $5 \mathrm{MeV}$ has been chosen because it is small enough to allow for conclusions for the $T=0$ case. Nevertheless, this temperature is large enough to allow for a smooth numerical representation of the spectral functions and to avoid the possibility of proton-neutron pairing instabilities [24-26]. In the next section, we briefly describe some specific features of the SCGF formalism for asymmetric nuclear matter. The discussion of the results and a systematic comparison with results of the Brueckner-Hartree-Fock approach is presented 
in Sec. III. The main conclusions are summarized in the last section.

\section{FORMALISM}

A key quantity in the theory of Green's functions-which allows us to compute all single-particle observables as well as the internal energy of the system-is the spectral function $A(k, \omega)$. It can be obtained as a solution of Dysons's equation,

$$
A_{\tau}(k, \omega)=\frac{-2 \operatorname{Im} \Sigma_{\tau}(k, \omega+\mathrm{i} \eta)}{\left[\omega-\frac{k^{2}}{2 m}-\operatorname{Re} \Sigma_{\tau}(k, \omega)\right]^{2}+\left[\operatorname{Im} \Sigma_{\tau}(k, \omega+\mathrm{i} \eta)\right]^{2}} .
$$

where $\Sigma_{\tau}(k, \omega+\mathrm{i} \eta)$ is the retarded self-energy of a nucleon with isospin projection $\tau$, which can either be a proton $(\tau=$ $+\frac{1}{2}$ or $p$ ) or a neutron $\left(\tau=-\frac{1}{2}\right.$ or $\left.n\right)$.

In the ladder or $T$-matrix approximation, the self-energy contains an energy-independent Hartree-Fock part and a complex dispersive contribution that accounts for correlations among the particles. The Hartree-Fock contribution to the ladder self-energy of a nucleon $\tau$ involves an explicit sum over the isospin projection $\tau^{\prime}$ of the internal particle,

$$
\begin{aligned}
\Sigma_{\tau}^{\mathrm{HF}}(k)= & \frac{1}{4 \pi} \sum_{\tau^{\prime}} \sum_{\substack{J S L \\
T \leqslant\left|\tau+\tau^{\prime}\right|}}(2 J+1)\left|C_{\frac{1}{2} \tau \frac{1}{2} \tau^{\prime}}^{T \tau+\tau^{\prime}}\right|^{2} \\
& \times \int \frac{\mathrm{d}^{3} k^{\prime}}{(2 \pi)^{3}}\left\langle q\left|V_{L L}^{J S T}\right| q\right\rangle n_{\tau^{\prime}}\left(k^{\prime}\right),
\end{aligned}
$$

with $V_{L L^{\prime}}^{J S T}$ the nuclear two-body potential in a partial wave representation. The relative momentum between the interacting particles is given by $\mathbf{q}=\frac{1}{2}\left(\mathbf{k}-\mathbf{k}^{\prime}\right)$ and the Clebsch-Gordan coefficient for the isospin quantum numbers is denoted by $C_{\frac{1}{2} \tau \frac{1}{2} \tau^{\prime}}^{T \tau+\tau^{\prime}}$. The momentum distribution,

$$
n_{\tau}(k)=\int_{-\infty}^{+\infty} \frac{\mathrm{d} \omega}{2 \pi} A_{\tau}(k, \omega) f_{\tau}(\omega)
$$

must be derived from the nontrivial spectral function. The Fermi-Dirac function for nucleons with isospin projection $\tau$ is denoted by $f_{\tau}(\omega)=\left\{\exp \left[\beta\left(\omega-\mu_{\tau}\right)\right]+1\right\}^{-1}$, where $\beta$ stands for the inverse temperature $T^{-1}$. For a given total density $\rho$, the partial fraction $x_{\tau}=\rho_{\tau} / \rho$ of the respective particle species is given by

$$
x_{\tau}=\frac{\gamma}{\rho} \int \frac{\mathrm{d}^{3} k}{(2 \pi)^{3}} n_{\tau}(k) \text {, }
$$

where $\gamma=2$ is the spin degeneracy factor of the system. The partial fractions of protons and neutrons add up to one $\left(x_{p}+x_{n}=1\right)$ and the asymmetry is given by $\alpha=x_{n}-x_{p}$. By considering a fixed composition, Eq. (5) can be used to fix the chemical potential $\mu_{\tau}$.

The generalized expression for the imaginary part of the self-energy in the $T$-matrix approximation for the case of asymmetric nuclear matter reads

$$
\begin{aligned}
\operatorname{Im} \Sigma_{\tau}(k, \omega+\mathrm{i} \eta)= & \frac{1}{4 \pi} \sum_{\tau^{\prime}} \sum_{\substack{J S L \\
T \leqslant\left|\tau+\tau^{\prime}\right|}}(2 J+1)\left|C_{\frac{1}{2} \tau \frac{1}{2} \tau^{\prime}}^{T \tau+\tau^{\prime}}\right|^{2} \\
& \times \int \frac{\mathrm{d}^{3} k^{\prime}}{(2 \pi)^{3}} \int_{-\infty}^{+\infty} \frac{\mathrm{d} \omega^{\prime}}{2 \pi} A_{\tau^{\prime}}\left(k^{\prime}, \omega^{\prime}\right) \\
& \times\left\langle q\left|\operatorname{Im} T_{L L}^{J S T \tau+\tau^{\prime}}\left(P, \omega+\omega^{\prime}+\mathrm{i} \eta\right)\right| q\right\rangle \\
& \times\left[f_{\tau^{\prime}}\left(\omega^{\prime}\right)+b_{\tau, \tau^{\prime}}\left(\omega+\omega^{\prime}\right)\right],
\end{aligned}
$$

where $b_{\tau, \tau^{\prime}}(\Omega)=\left\{\exp \left[\beta\left(\Omega-\mu_{\tau}-\mu_{\tau^{\prime}}\right)\right]-1\right\}^{-1}$ is the Bose distribution function. The total pair momentum is given by $P=\frac{1}{2}\left|\mathbf{k}+\mathbf{k}^{\prime}\right|$. A dispersion relation that is reported, for example, in Ref. [16] determines the real part of the dispersive contribution to the ladder self-energy.

The $T$-matrix elements contain the resummation of the ladder diagrams to all orders and can be obtained as the solution of a scattering-type integral equation,

$$
\begin{aligned}
& \left\langle q\left|T_{L L^{\prime}}^{J S T m_{T}}(P, \Omega+\mathrm{i} \eta)\right| q^{\prime}\right\rangle \\
& =\left\langle q\left|V_{L L^{\prime}}^{J S T}\right| q^{\prime}\right\rangle+\sum_{L^{\prime \prime}} \int_{0}^{\infty} \frac{\mathrm{d} k^{\prime} k^{\prime 2}}{(2 \pi)^{3}}\left\langle q\left|V_{L L^{\prime \prime}}^{J S T}\right| k^{\prime}\right\rangle \\
& \quad \times \bar{g}_{m_{T}}^{\mathrm{II}}\left(P, \Omega+\mathrm{i} \eta, k^{\prime}\right)\left\langle k^{\prime}\left|T_{L^{\prime \prime} L^{\prime}}^{J S T m_{T}}(P, \Omega+\mathrm{i} \eta)\right| q^{\prime}\right\rangle .
\end{aligned}
$$

Since in ANM, the in-medium propagation of a neutron is different from that of a proton, the $T$-matrix elements depend on the third component of the isospin of the propagating pair, $m_{T}=\tau+\tau^{\prime}$, via the noninteracting two-particle propagator $g_{m_{T}}^{\mathrm{II}}$,

$$
\begin{aligned}
g_{\tau_{1}+\tau_{2}}^{\mathrm{II}}\left(k_{1}, k_{2}, \Omega+\mathrm{i} \eta\right)= & \int_{-\infty}^{+\infty} \frac{\mathrm{d} \omega}{2 \pi} \int_{-\infty}^{+\infty} \frac{\mathrm{d} \omega^{\prime}}{2 \pi} A_{\tau_{1}}\left(k_{1}, \omega\right) \\
& \times A_{\tau_{2}}\left(k_{2}, \omega^{\prime}\right) \frac{1-f_{\tau_{1}}(\omega)-f_{\tau_{2}}\left(\omega^{\prime}\right)}{\Omega-\omega-\omega^{\prime}+\mathrm{i} \eta} .
\end{aligned}
$$

To circumvent the coupling between partial waves of different total angular momenta, $g_{m_{T}}^{\text {II }}$ enters Eq. (7) in an angle-averaged form that is indicated by the bar.

Equations (2)-(8) must be solved self-consistently for a given temperature $\beta^{-1}$, a given density $\rho$, and a given proton fraction $x_{p}$. Partial waves up to $J=9$ were included for the calculation of the energy-independent part of the self-energy. The dispersive part includes only partial waves up to $J=2$. A more comprehensive description of the numerical details is given in Ref. [16]. The numerical routine that was developed for ANM was tested in the following way: The results for SNM and PNM are recovered if one chooses $x_{p}=0.5$ and $x_{p}=0$, respectively. Furthermore, we have checked that charge symmetry is fulfilled, which can be expressed by the condition $\Sigma_{p}\left(k, \omega, x_{p}\right)=\Sigma_{n}\left(k, \omega, 1-x_{p}\right)$.

Once the self-consistent solution for the spectral function has been obtained, the internal energy per particle in the system 


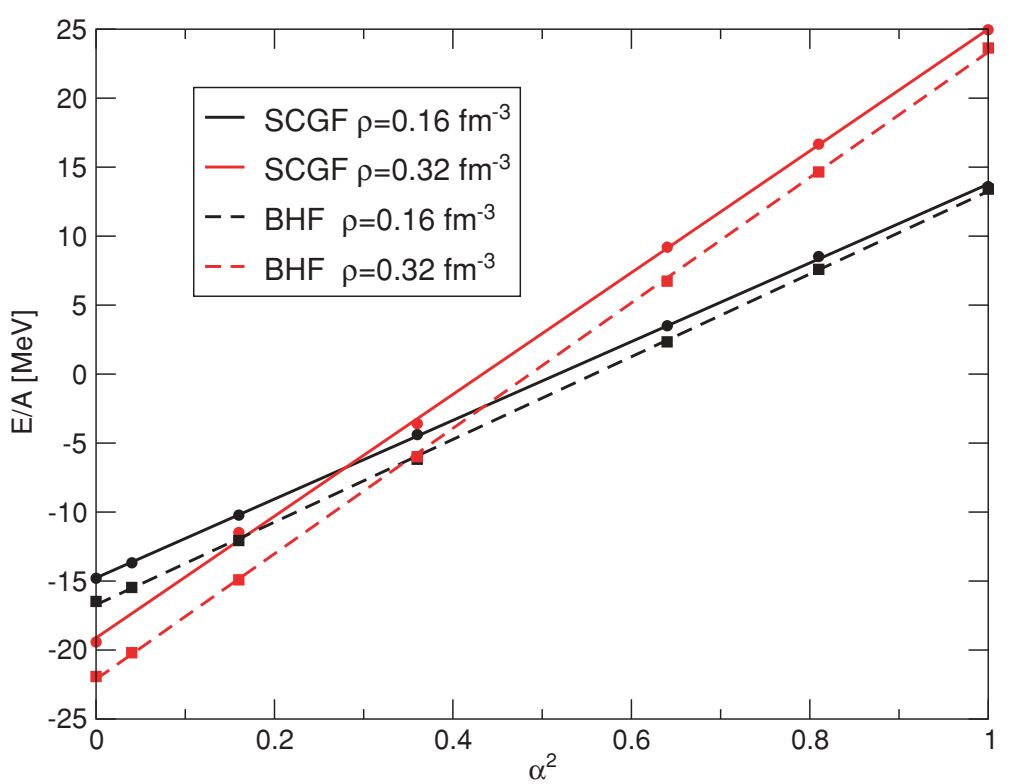

FIG. 1. (Color online) Dependence of $E / A$ on $\alpha^{2}$ computed in the SCGF approach (full lines) and in the BHF approach (dashed lines) at two densities, $\rho=$ $0.16 \mathrm{fm}^{-3}$ (black lines) and $\rho=0.32 \mathrm{fm}^{-3}$ (red lines). is given by

$\frac{E}{A}=\frac{\gamma}{\rho} \sum_{\tau} \int \frac{\mathrm{d}^{3} k^{\prime}}{(2 \pi)^{3}} \int_{-\infty}^{+\infty} \frac{\mathrm{d} \omega^{\prime}}{2 \pi} \frac{1}{2}\left(\frac{k^{2}}{2 m}+\omega\right) A_{\tau}(k, \omega) f_{\tau}(\omega)$.

The BHF approach is obtained from the previous formulation by assuming that the single-particle spectral functions are characterized by only one energy having the full strength accumulated in this energy, $A_{\tau}(k, \omega)=\delta\left(\omega-\epsilon_{\tau}^{\mathrm{BHF}}(k)\right)$, with $\epsilon_{\tau}^{\mathrm{BHF}}(k)$ the BHF single-particle energy. In addition, in the twobody propagator [Eq. (8)] one only considers the propagation of particle states. Notice also that the BHF self-energy does not include the contribution of the Bose distribution that appears in Eq. (6).

\section{RESULTS AND DISCUSSION}

All the results discussed in this paper have been computed for the charge-dependent Bonn (CDBONN) potential, defined in [27], which is nonlocal and exhibits a softer tensor component compared to other realistic potentials such as the Argonne V18 [28] or Reid93 [29], which are local. Since we want to concentrate on the asymmetry dependence, we will only consider one temperature $T=5 \mathrm{MeV}$, low enough for the conclusions on the asymmetry dependence to be valid at $T=$ $0 \mathrm{MeV}$ and high enough to avoid the instabilities associated with neutron-proton pairing.

The binding energies of asymmetric nuclear matter calculated in the BHF approximation and within the framework of the SCGF theory are reported in Fig. 1 as a function of the square of the asymmetry parameter $\alpha$. The plots correspond to two densities, the empirical saturation density of symmetric nuclear matter, $\rho=0.16 \mathrm{fm}^{-3}$, and twice this density. Notice, however, that BHF calculations using the CDBONN potential yield a saturation point at higher densities.

The first thing to realize is the linear dependence of the energy in terms of $\alpha^{2}$, for both types of calculations (BHF and SCGF) in the full range of variation of asymmetry from SNM to PNM. In the case of BHF, this fact has already been considered in the literature [21] and provides the justification for using PNM and SNM to determine the coefficients in Eq. (1), which turns out to be a very good approximation.

The propagation of holes in the SCGF and the consideration of the spectral functions in the intermediate states of the ladder equation result in a repulsive effect with respect to the continuous-choice BHF calculation. This repulsive effect increases with density, being 1.8 and $3.2 \mathrm{MeV}$ in SNM matter at $\rho=0.16 \mathrm{fm}^{-3}$ and $\rho=0.32 \mathrm{fm}^{-3}$, respectively.

However, for a given density, the difference between the SCGF and the BHF calculations does not depend so much on the asymmetry, which is slightly greater in SNM than in PNM. This leads to a small decrease of the symmetry energy of the SCGF calculation compared to the BHF one, which is also calculated at $T=5 \mathrm{MeV}$. At $\rho=0.16 \mathrm{fm}^{-3}, a_{s}=30.0 \mathrm{MeV}$ in the BHF approximation whereas the SCGF schemes provide $a_{s}=28.6 \mathrm{MeV}$. This is in qualitative agreement with a recent comparison of both approaches [17], in which the symmetry energy has been deduced from SNM and PNM calculations. The reduction of the symmetry obtained in the present analysis, however, is weaker than the decrease reported in [17] by around $4 \mathrm{MeV}$. In this reference, however, the Reid93 potential, which has a stronger tensor component and also stronger short-range correlations than the CDBONN, was employed. Furthermore it should be noted that the spectral functions employed in [17] are described in terms of three $\delta$ functions, whereas a continous description has been used in the present approach. In addition, the calculation of [17] was performed at $T=0$ whereas we consider $T=5 \mathrm{MeV}$. We do not believe, however, that the temperature can be the origin of this discrepancy. In fact, if one considers the model of a noninteracting Fermi gas, one finds that the symmetry energy at normal density $\rho_{0}$ is reduced by $0.5 \mathrm{MeV}$ going from $T=0$ to the case of $T=5 \mathrm{MeV}$.

In discussing the differences for the symmetry energy between SCGF and BHF one must keep in mind that the loss of 


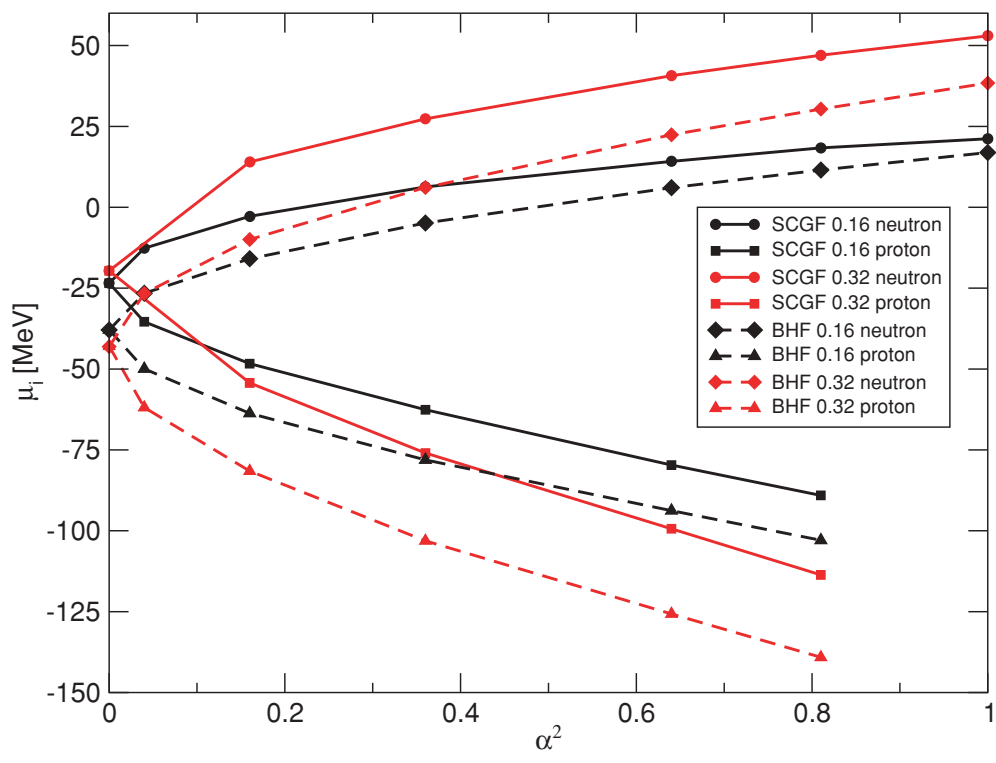

FIG. 2. (Color online) Dependence of neutron (upper curves) and proton (lower curves) chemical potentials $\mu$ on the asymmetry $\alpha$ calculated in the SCGF approach (solid lines) and in the BHF approach (dashed curves) at two given densities, $\rho=0.16 \mathrm{fm}^{-3}$ (black lines) and $\rho=0.32 \mathrm{fm}^{-3}$ (red lines). energy in SCGF compared to BHF results from a delicate balance between an increase of kinetic energy and more attractive potential energy. In fact, the perturbative inclusion of the holehole scattering terms of [23] even leads to an increase of the symmetry energy.

The second point that we want to consider is the dependence of the chemical potential of protons and neutrons on the asymmetry. Figure 2 shows the chemical potential of neutrons (upper curves) and protons (lower curves) calculated in the SCGF approach (solid lines) and in the BHF approach (dashed curves). The chemical potential of protons and neutrons coincide at the symmetric case $(\alpha=0)$, as we have not considered the charge symmetry breaking terms contained in the CDBONN potential. When the asymmetry increases, the neutron chemical potential increases and becomes positive, whereas the protons potential becomes more and more attractive. The dependence on $\alpha^{2}$ is no longer linear. One should keep in mind that in the case of SCGF, the chemical potential that one obtains from the normalization condition of the partial density should coincide with the one obtained from the free energy using the thermodynamic relation $\mu_{\tau}=F / A+\rho \frac{\partial F / A}{\partial \rho_{\tau}}$. In contrast, it is well known that in the BHF approach the chemical potential derived from the normalization condition substantially differs from the one obtained by the thermodynamic relation. Actually, this is already true at $T=0 \mathrm{MeV}$ where one needs to incorporate the rearrangement terms in the self-energy to recover the relation $\mu=\epsilon\left(k_{f}\right)$ (where $\epsilon\left(k_{F}\right)$ is the single-particle energy at the Fermi surface).

It is important to note that the differences between the BHF and the SCGF chemical potentials are much larger (of $\sim 15 \mathrm{MeV}$ in the whole range of asymmetries) than the differences in the energy per particle discussed in Fig. 1, thus indicating that the role of correlations has a greater influence in this observable than in the energy per particle and also giving an idea of the magnitude of the rearrangement term in such calculations. In particular we want to emphasize that the difference between the chemical potentials for neutrons and protons is larger for BHF than it is for SCGF. This implies that the SCGF tends to predict $\beta$ equilibrium with a smaller proton fraction as derived from $\mathrm{BHF}$ calculations.

At this point we want to remark again that the results discussed in this work (BHF as well as SCGF) have been obtained for a finite temperature of $T=5 \mathrm{MeV}$. The chemical potentials displayed in Fig. 2, in particular the ones for large asymmetries, deviate from those calculated at zero temperature. To have a simple estimate of this temperature dependence we consider again the Fermi-gas model of noninteracting nucleons and find, at density $\rho_{0}$ and asymmetry $\alpha=0.9$, the chemical potential of the neutron to be smaller by $0.4 \mathrm{MeV}$ at $T=5 \mathrm{MeV}$ compared to that at $T=0$, whereas the chemical potential for the protons is smaller by even $3 \mathrm{MeV}$ at $T=5 \mathrm{MeV}$ compared to the case of $T=0$. This is because at large asymmetries and finite temperature, the minority species can be far from the degenerate limit and its chemical potential can be very different from the zero-temperature value. Therefore, caution should be taken in the extrapolation of chemical potentials at zero temperature when asymmetries are large.

The next point we shall address is the discussion of the single-particle spectral functions. Figure 3 shows the proton spectral functions at $\rho=0.16 \mathrm{fm}^{-3}$ for the momentum $k=0 \mathrm{MeV}$ at different proton fractions as a function of the energy measured with respect to the proton chemical potential corresponding to each fraction. As the asymmetry increases, the amount of protons decreases and the Fermi momentum for the protons gets closer to $k=0 \mathrm{MeV}$. As a consequence, the coupling to two-hole one-particle configurations with this momentum of $k=0$ is reduced, with the quasi-particle peak getting narrower and higher. The spectral function at positive energies, however, is larger with increasing asymmetry. This can be explained by the fact that correlation effects mainly originate from the tensor force, which is more important in proton-neutron interactions than in neutron-neutron or proton-proton interactions. Note that the most important partial wave for these tensor correlations is the ${ }^{3} S_{1}-{ }^{3} D_{1}$ channel, which is relevant for nucleon pairs with isospin 


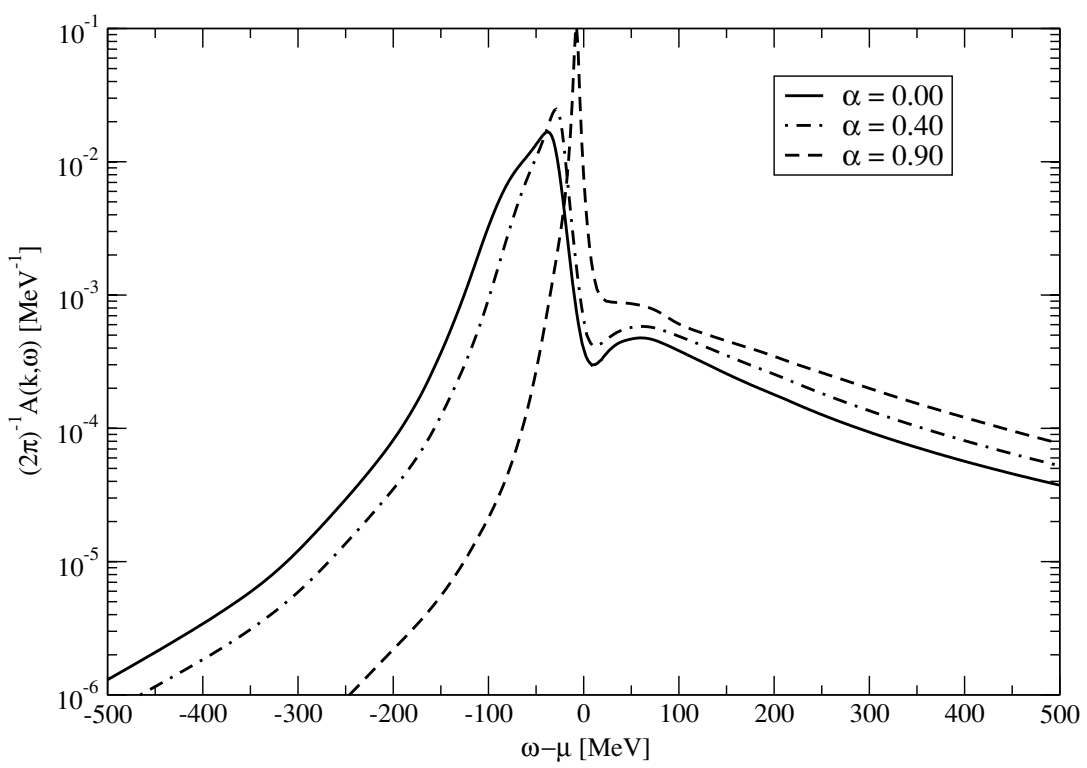

FIG. 3. $k=0 \mathrm{MeV}$ proton spectral function for different proton fractions. The calculations were performed at $\rho=0.16 \mathrm{fm}^{-3}$ and $T=5 \mathrm{MeV}$.

zero only. Since the density of neutrons increases with the asymmetry parameter, the protons display stronger correlation effects at these larger asymmetries leading to an enhancement for the spectral function for $k<k_{F}$ at energies $\omega>\mu$.

The corresponding plot for the neutron spectral functions is presented in Fig. 4. Again we consider the momentum $k=0$, the same density $\rho=0.16 \mathrm{fm}^{-3}$, and asymmetries. Looking at the spectral functions at energies above the chemical potential for the neutrons, we can see that the correlations that are responsible for the spectral function in this regime are reduced with the asymmetry, that is, with the density of protons. This can again be understood from the dominance of the proton-neutron interaction leading to these correlations. The width of the neutron spectral functions seems not to be affected very much by an increase of the asymmetry for the considered values of $\alpha$. This results from the damping of the strong isospin-zero correlations at larger values of $\alpha$ being counterbalanced by an increasing phase space for the neutron-neutron configurations. Similar observations have also been made in the perturbative calculations of asymmetric matter in [23].

To explore a bit more the width and height of the quasiparticle peak in the spectral function and its dependence on the asymmetry we show in Fig. 5 the width of the spectral function given by $2\left|\operatorname{Im} \Sigma\left(k, \epsilon_{\mathrm{qp}}\right)\right|$, where $\epsilon_{\mathrm{qp}}$ is the quasi-particle energy. Obviously, for the symmetric case both the widths for protons and neutrons coincide. When the asymmetry increases, the width of the protons decreases monotonically, indicating that $k=0$ is getting closer and closer to the proton Fermi surface. The situation for the neutrons is a little bit different. For low asymmetries, the width initially increases with the asymmetry, reaches a maximum, and then it decreases again-being a little lower for pure neutron matter than for the symmetric case. This indicates that there is an asymmetry

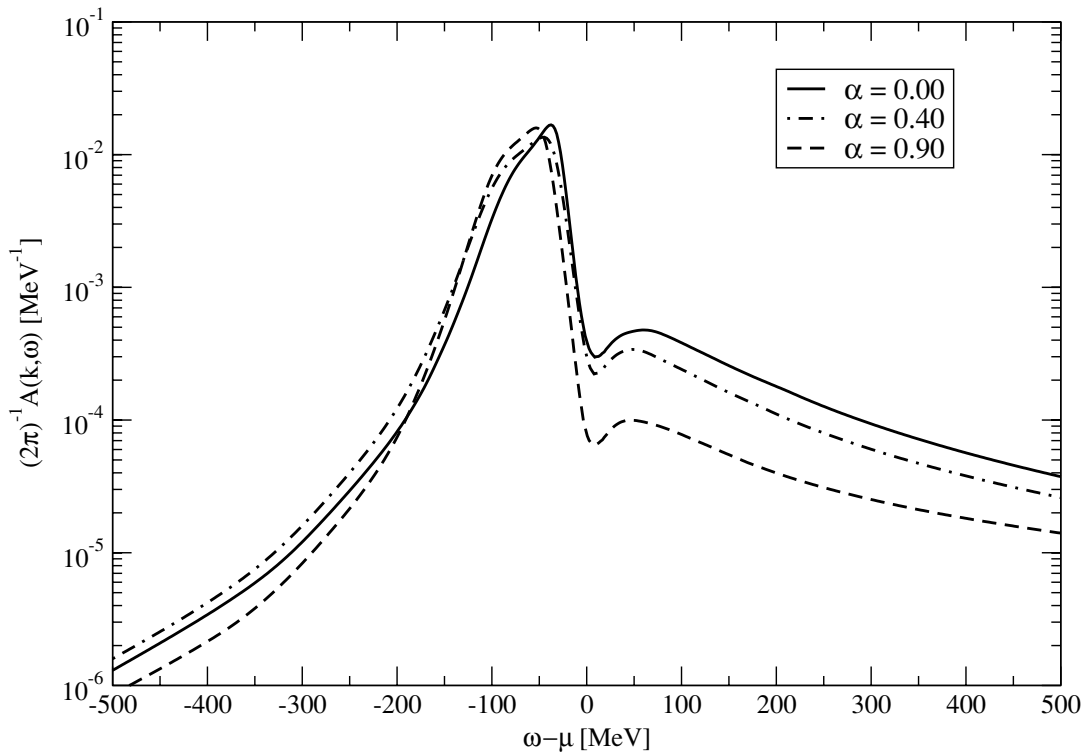

FIG. 4. $k=0 \mathrm{MeV}$ neutron spectral function for different proton fractions. The calculations were performed at $\rho=0.16 \mathrm{fm}^{-3}$ and $T=$ $5 \mathrm{MeV}$. 


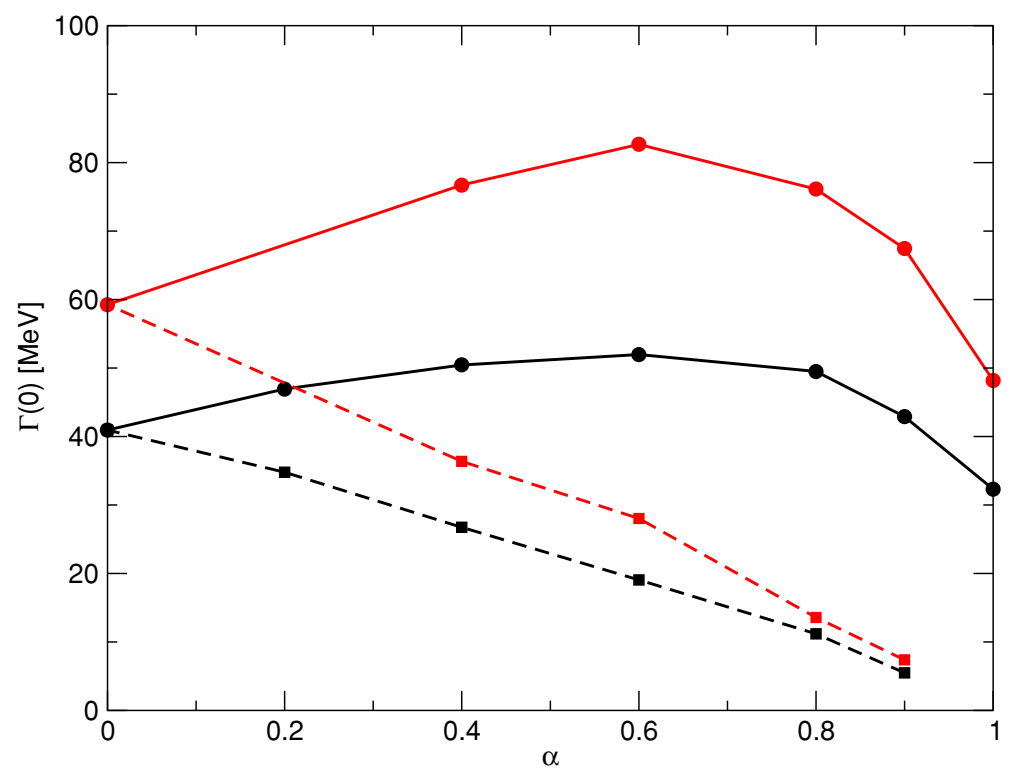

FIG. 5. (Color online) Dependence of the $k=$ $0 \mathrm{MeV}$ on-shell width $\Gamma(0)$ on the proton fraction for neutrons (full lines) and protons (dashed lines) at two given densities, $\rho=0.16 \mathrm{fm}^{-3}$ (black lines) and $\rho=0.32 \mathrm{fm}^{-3}$ (red lines).

for which the correlation effects on the width of the neutron quasi-particle peak reach a maximum. As already mentioned, there is a competition between the correlations originated by the propagation of neutron-neutron holes, which should increase when the asymmetry gets larger as the associated phase space also grows, and the correlations originating from the interaction of the neutrons with the protons. It is clear that these last correlations get less important for the neutrons with increasing asymmetry. Finally, for neutron matter, the decrease of the number of protons and therefore the suppression of the tensor components of the neutron-proton interaction dominates and the width for neutron matter is slightly smaller than for symmetric nuclear matter.

Another observable that has been the object of discussion during the past few years, owing to its relevance in the analysis of the $\left(e, e^{\prime} p\right)$ reaction, is the momentum distribution, which also provides a clear measurement of the effects of correlations. In Fig. 6, we show the occupation of the zero-momentum state as a function of the asymmetry. In the symmetric case, we can observe an unexpected behavior on the occupation as a function of density: At the higher density there is a higher occupation whereas at the lower one the occupation is smaller, a behavior that has been pointed out previously in the literature [8]. For a given density, proton depletion increases with asymmetry, indicating the importance of the neutron-proton correlations. In contrast, the depletion of the neutrons gets smaller, that is, neutron matter is a less correlated system. Also it is worth noting that for pure neutron matter one recovers the expected behavior of having a higher occupation for smaller density.

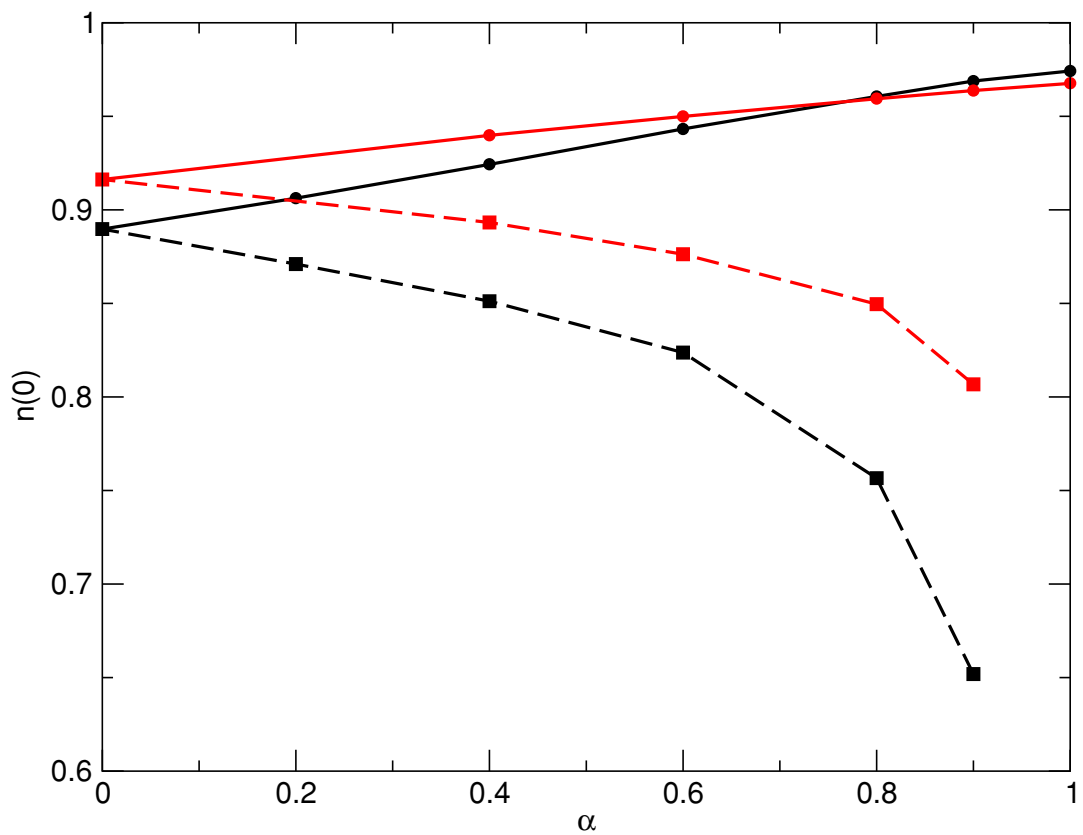

FIG. 6. (Color online) Dependence of the $n(0)$ on the proton fraction for neutrons (full lines) and protons (dashed lines) at two given densities, $\rho=0.16 \mathrm{fm}^{-3}$ (black lines) and $\rho=$ $0.32 \mathrm{fm}^{-3}$ (red lines). 


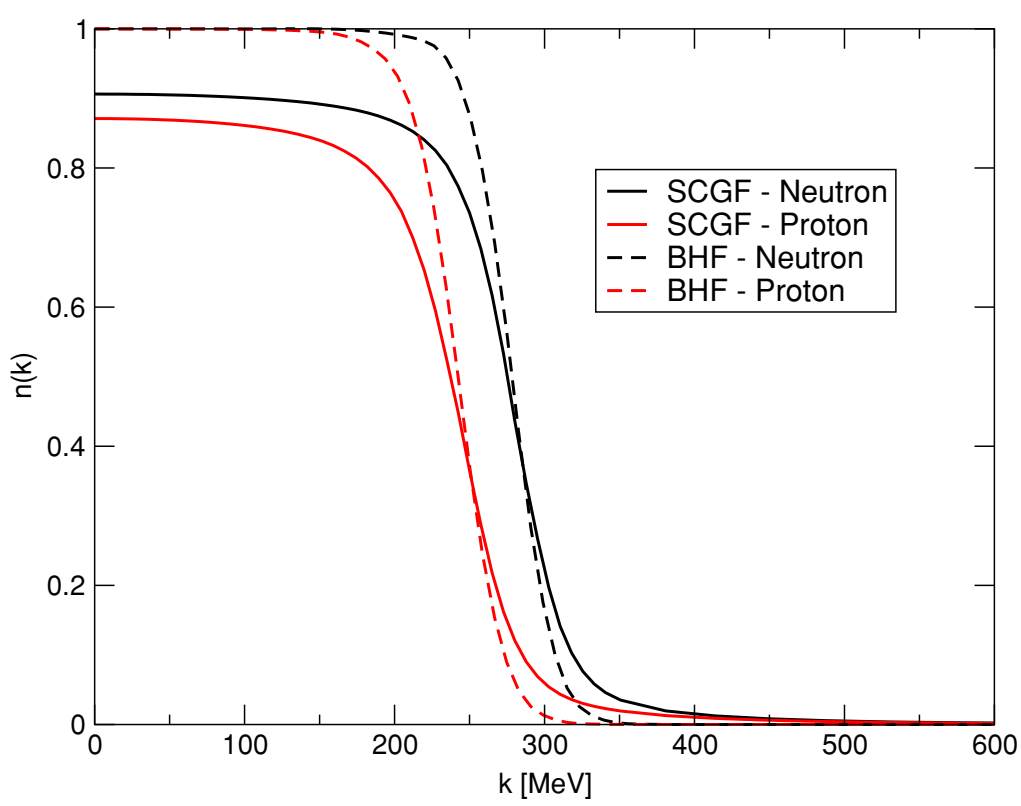

FIG. 7. (Color online) Momentum distributions for neutrons and protons for the SCGF approach (full lines) and the BHF approach (dashed lines) at a density $\rho=0.16 \mathrm{fm}^{-3}$ and asymmetry $\alpha=0.2$.
Finally, in Fig. 7, we show the momentum distribution at $\rho=0.16 \mathrm{fm}^{-3}$ and an asymmetry $\alpha=0.2$, which is characteristic of heavy nuclei, such as ${ }^{208} \mathrm{~Pb}$. Included in the figure are also the BHF momentum distributions. The first thing to notice is that the BHF momentum distributions do not contain correlation effects and are very similar to a normal thermal Fermi distribution. The momentum distributions obtained within the framework of the SCGF contain, besides thermal effects, important short-range and tensor correlations, which are reflected in the depletion of the occupation at low momentum and in a larger occupation than the BHF momentum distribution at large momenta. Notice also that the proton momentum distribution is more depleted than the neutron momentum distribution. This is in agreement with all the previous discussions, reflecting the fact that the protons (i.e., the less abundant particle in the realistic asymmetric conditions) are more affected by correlations, mainly due to their interactions with neutrons. This may be important in the interpretation of the momentum distributions obtained in $\left(e, e^{\prime} p\right)$ experiments. Some of the analysis has been conducted within the framework of a local density approximation starting from results obtained in SNM, where the momentum distribution of protons and neutrons are identical. This different behavior of the momentum distributions of protons and neutrons contrasts with the very recent calculations by Bożek [20] also obtained within the SCGF framework and with the CDBONN potential in which no noticeable difference between the momentum distributions of neutrons and protons was found.

\section{SUMMARY AND CONCLUSIONS}

The techniques to evaluate the single-particle Green's function in a self-consistent $T$-matrix approach (SCGF), which has recently been developed for nuclear matter [16], have been extended and applied to asymmetric matter. Actual calculations have been performed using the realistic CDBONN interaction for asymmetric matter at two densities (the saturation density of symmetric nuclear matter and twice this density), at various asymmetries, and for a temperature of $T=5 \mathrm{MeV}$. This temperature is low enough to allow for conclusions on the $T=0$ limit and high enough to avoid the instabilities associated to neutron-proton pairing.

The inclusion of the hole-hole ladders and the selfconsistent treatment of the Green's function in the SCGF approach leads to a small reduction of the binding energy per nucleon compared to the BHF approximation. This effect increases with density and is slightly weaker for pure neutron matter compared to symmetric nuclear matter, which leads to a small reduction in the symmetry energy.

Larger effects are observed for the single-particle properties such as the chemical potential. In particular we observe in neutron-rich matter a reduction in the difference between the chemical potentials for protons and neutrons, which would correspond to smaller proton fractions in $\beta$-stable matter than those predicted by a BHF-type equation of state.

The SCGF calculation also yields detailed informations on the single-particle spectral functions and momentum distributions for protons and neutrons. We observe a depletion of the proton occupancies for momenta below the Fermi momentum, which increases significantly with neutron fraction. This can be explained by the strong correlations induced from protonneutron interactions.

\section{ACKNOWLEDGMENTS}

Useful discussions with Dr. Isaac Vidaña are gratefully acknowledged. This work has been supported by the Europäische Graduiertenkolleg Tübingen-Basel (DFG-SNF), the German-Spanish exchange program (DAAD, Aciones Integradas Hispano-Alemanas), the DGICYT (Spain) Project No. BFM2002-01868, and the Generalitat de Catalunya Project No. 2001SGR00064. 
[1] H. Heiselberg and M. Hjorth-Jensen, Phys. Rep. 328, 237 (2000).

[2] M. Baldo, Nuclear Methods and the Nuclear Equation of State, Int. Rev. of Nucl. Phys, Vol. 9 (World Scientific, Singapore, 1999).

[3] H. Müther and A. Polls, Prog. Part. Nucl. Phys. 45, 243 (2000).

[4] W. H. Dickhoff and C. Barbieri, Prog. Part. Nucl. Phys. 52, 377 (2004).

[5] M. F. van Batenburg, Ph.D. thesis, University of Utrecht, 2001.

[6] A. Ramos, A. Polls, and W. H. Dickhoff, Nucl. Phys. A503, 1 (1989).

[7] O. Benhar, A. Fabrocini, and S. Fantoni, Nucl. Phys. A505, 267 (1989).

[8] H. Müther, G. Knehr, and A. Polls, Phys. Rev. C 52, 2955 (1995).

[9] B. D. Day, Rev. Mod. Phys. 39, 719 (1967).

[10] A. Akmal and V. R. Pandhariapnde, Phys. Rev. C 56, 2261 (1997).

[11] S. Fantoni and A. Fabrocini, in Microscopic Quantum ManyBody Theories and Their Applications, edited by J. Navarro and A. Polls (Springer, New York, 1998).

[12] S. Fantoni, in Introduction to Modern Methods of Quantum Many-Body Theory and Their Applications, edited by A. Fabrocini, S. Fantoni, and E. Krotscheck, Series on Advances in Quantum Many-Body Theory, Vol. 7 (World Scientific, Singapore, 2002).

[13] W. D. Kraeft, D. Kremp, W. Ebeling, and G. Röpke, Quantum Statistics of Charged Particle Systems (Akademie-Verlag, Berlin, 1986).
[14] L. P. Kadanoff and G. Baym, Quantum Statistical Mechanics (Benjamin, New York, 1962).

[15] Y. Dewulf, W. H. Dickhoff, D. Van Neck, E. R. Stoddard, and M. Waroquier, Phys. Rev. Lett. 90, 152501 (2003).

[16] T. Frick and H. Müther, Phys. Rev. C 68, 034310 (2003).

[17] A. E. L. Dieperink, Y. Dewulf, D. Van Neck, M. Waroquier, and V. Rodin, Phys. Rev. C 68, 064307 (2003).

[18] P. Bożek, Phys. Rev. C 59, 2619 (1999).

[19] P. Bożek, Phys. Rev. C 65, 054306 (2002).

[20] P. Bożek, Phys. Lett. B586, 239 (2004).

[21] W. Zuo, I. Bombaci, and U. Lombardo, Phys. Rev. C 60, 024605 (1999).

[22] I. Vidaña and I. Bombaci, Phys. Rev. C 66, 045801 (2002).

[23] Kh. S. A. Hassaneen and H. Müther, Phys. Rev. C 70, 054308 (2004).

[24] B. E. Vonderfecht, W. H. Dickhoff, A. Polls, and A. Ramos, Nucl. Phys. A555, 1 (1993).

[25] T. Alm, G. Röpke, A. Schnell, N. H. Kwong, and H. S. Köhler, Phys. Rev. C 53, 2181 (1996).

[26] P. Bożek, Nucl. Phys. A657, 187 (1999).

[27] R. Machleidt, F. Sammarruca, and Y. Song, Phys. Rev. C 53 R1483 (1996).

[28] R. B. Wiringa, V. G. J. Stoks, and R. Schiavilla, Phys. Rev. C 51, 38(1995).

[29] V. G. J. Stoks, R. A. M. Klomp, C. P. F. Terhegen, and J. J. de Swart, Phys. Rev. C 49, 2950 (1994). 